\title{
Far infra-red therapy promotes ischemia-induced angiogenesis in diabetic mice and restores high glucose-suppressed endothelial progenitor cell functions
}

\author{
Po-Hsun Huang ${ }^{1,5,6}$, Jaw-Wen Chen ${ }^{1,2,6,7^{*}}$, Chih-Pei Lin ${ }^{3,8}$, Ying-Hwa Chen ${ }^{1,6}$, Chao-Hung Wang ${ }^{5,9}$,
} Hsin-Bang Leu ${ }^{1,4,5,6}$ and Shing-Jong Lin ${ }^{1,2,5,6^{*}}$

\begin{abstract}
Background: Far infra-red (IFR) therapy was shown to exert beneficial effects in cardiovascular system, but effects of IFR on endothelial progenitor cell (EPC) and EPC-related vasculogenesis remain unclear. We hypothesized that IFR radiation can restore blood flow recovery in ischemic hindlimb in diabetic mice by enhancement of EPCS functions and homing process.
\end{abstract}

Materials and methods: Starting at 4 weeks after the onset of diabetes, unilateral hindlimb ischemia was induced in streptozotocine (STZ)-induced diabetic mice, which were divided into control and IFR therapy groups ( $n=6$ per group). The latter mice were placed in an IFR dry sauna at $34^{\circ} \mathrm{C}$ for 30 min once per day for 5 weeks.

Results: Doppler perfusion imaging demonstrated that the ischemic limb/normal side blood perfusion ratio in the thermal therapy group was significantly increased beyond that in controls, and significantly greater capillary density was seen in the IFR therapy group. Flow cytometry analysis showed impaired EPCS $\left(\mathrm{Sca}-1^{+} / \mathrm{Flk}-1^{+}\right)$mobilization after ischemia surgery in diabetic mice with or without IFR therapy ( $n=6$ per group). However, as compared to those in the control group, bone marrow-derived EPCs differentiated into endothelial cells defined as $\mathrm{GFP}^{+} / \mathrm{CD} 31^{+}$ double-positive cells were significantly increased in ischemic tissue around the vessels in diabetic mice that received IFR radiation. In in-vitro studies, cultured EPCs treated with IFR radiation markedly augmented high glucose-impaired EPC functions, inhibited high glucose-induced EPC senescence and reduced $\mathrm{H}_{2} \mathrm{O}_{2}$ production. Nude mice received human EPCS treated with IFR in high glucose medium showed a significant improvement in blood flow recovery in ischemic limb compared to those without IFR therapy. IFR therapy promoted blood flow recovery and new vessel formation in STZ-induced diabetic mice.

Conclusions: Administration of IFR therapy promoted collateral flow recovery and new vessel formation in STZ-induced diabetic mice, and these beneficial effects may derive from enhancement of EPC functions and homing process.

Keywords: Far infra-red therapy, Endothelial progenitor cell, Diabetes, Ischemia

\footnotetext{
* Correspondence: jwchen@vghtpe.gov.tw; sjlin@vghtpe.gov.tw

'Division of Cardiology, Taipei Veterans General Hospital, Taipei, Taiwan ${ }^{2}$ Department of Medical Research and Education, Taipei Veterans General Hospital, No. 201, Sec. 2, Shih-Pai Road, Taipei, Taiwan

Full list of author information is available at the end of the article
} 


\section{Introduction}

Angiogenesis, defined as sprouting of blood vessels from preexisting vascular structures, is a physiological response to ischemia but often fails to produce sufficient collateral flow to rescue ischemic organ [1]. In animal models of ischemia, increasing evidence indicates that administration of angiogenic growth factors, either through recombinant protein or by gene transfer, can increase nutrients perfusion through neovascularization and reduce ischemia-related organ damage [2]. However, there is clinical evidence that some patients, unlike healthy experimental animals, fail to develop collateral circulation after tissue ischemia and appear to be refractory to administration of exogenous growth factors [3]. Patients with diabetes are related to endothelial dysfunction and have diminished ability for collateral vessel formation in response to ischemia in the heart and peripheral tissues [4-6].

Convincing evidence suggests that neovascularization in adults is not solely the result of proliferation of local endothelial cells (angiogenesis), but also involves bone marrow-derived circulating endothelial progenitor cells (EPCs) for the processes of vasculogenesis [7]. However, patients with cardiovascular risk factors were shown to have decreased numbers and functions of circulating EPCs [8]. Our recent study also demonstrated that long-term exposure to high glucose may enhance cellular senescence and decrease cell numbers and functional competencies of EPCs via nitric oxide (NO)related mechanisms [9]. Recent evidence showed that the circulating EPCs levels and arterial stiffness were closely related to their glycemic control in patients with type 2 diabetes mellitus (DM) [10]. These findings provide a rationale for potential therapeutic targets on hyperglycemia-related vascular complications in diabetic patients. Far infrared (IFR) radiation is an invisible electromagnetic wave with a characteristic wavelength between 5.6 and $1000 \mu \mathrm{m}$ that can be perceived as heat by thermo-receptors in the skin $[11,12]$. Recent studies indicate that IFR therapy exerts beneficial effects in the cardiovascular system. IFR radiation improves ventricular arrhythmias and endothelial function in patients with heart disease, and enhances access flow and patency of arteriovenous fistulas in hemodialysis patients [13-15]. In addition, IFR therapy promotes microvascular blood flow and angiogenesis in various animal models $[16,17]$. Although clinical studies have indicated that IFR radiation can exert beneficial effects in the cardiovascular system, the multi-faceted effects of IFR therapy on circulating EPCs and diabetes remain unclear. Therefore, we hypothesized that IFR radiation can improve blood flow recovery after tissue ischemia in diabetic mice and improve functional capacities of EPCs by increasing NO bioavailability.

\section{Materials and methods \\ Animals}

Mice at 6-8 weeks old were purchased from the National Laboratory Animal Center, Taiwan (FVB mice). Experimental diabetes was induced in FVB mice by daily intraperitoneal injections of streptozotocin (STZ) in citrate buffer $(40 \mathrm{mg} / \mathrm{kg})$ for 5 days for the type 1 diabetic model [18]. Mice were considered diabetic only if they developed glycemia $>250 \mathrm{mg} / \mathrm{dl}$ and overt glycosuria at 14 days after the first STZ injection. Persistence of diabetes was determined at the end of the study. All mice were kept in microisolator cages on a 12-h day/night cycle. The investigation conforms with the Guide for the Care and Use of Laboratory Animals published by the US National Institutes of Health (NIH Publication 1996). All experimental procedures and protocols involving animals were approved by the institutional animal care committee of National Yang-Ming University (Taipei, Taiwan) and in compliance with the ARRIVE guidelines [19].

\section{Mouse ischemic hindlimb model}

Eight-week-old male wild-type mice and STZ-induced diabetic mice received unilateral hindlimb ischemia surgery by excising the right femoral artery as previously described [20]. Local IFR therapy was given in STZinduced diabetic mice for $30 \mathrm{~min}$ twice per day for 2 weeks after the surgery, and wild-type and diabetic control mice were placed on a heating plate at $34^{\circ} \mathrm{C}$ for 30 min twice daily to avoid the thermal effect in this study. Briefly, mice were anaesthetized by an intraperitoneal injection of ketamine $(100 \mathrm{mg} / \mathrm{kg})$ and xylazine $(10 \mathrm{mg} / \mathrm{kg})$. The depth of anesthesia was checked by ensuring that noxious pinch stimulation (blunt forceps) of the hindpaw, the forepaw, and the ear did not evoke any motor reflexes. The proximal and distal portions of the femoral artery were ligated. Hindlimb blood perfusion was measured with a Laser Doppler perfusion imager system (Moor Instruments Limited, Devon, UK) before and after the surgery and followed weekly. To avoid the influence of ambient light and temperature, the results were expressed as the ratio of perfusion in the right (ischemic) versus left (non-ischemic) limb.

\section{Measurement of capillary density and oxidative stress in the ischemic limb}

At 4 weeks after surgery, the mice were euthanized via intravenous ketamine injection. The femora were carefully removed, and the ischemic thigh muscles were embedded in paraffin. Sections $(5 \mu \mathrm{m})$ were de-paraffinized and incubated with a rat monoclonal antibody against murine CD31 (clone MEC13.1, BD PharMingen, San Diego, CA). Antibody distribution was visualized by using the avidin-biotin-complex technique and Vector 
Red Chromogenic substrate (Vector Laboratories, Burlingame, CA), followed by counterstaining with hematoxylin. Capillaries were identified by positive staining for CD31. Ten different fields from each tissue preparation were randomly selected, and visible capillaries were counted. Capillary density was expressed as the number of capillaries per square millimeter. To evaluate local oxidative stress levels in ischemic muscles, an antibody against nitrotyrosine (Upstate, Lake Placid, NY, USA) was used.

\section{Flow cytometry}

To investigate the effects of IFR therapy on EPC mobilization in response to tissue ischemia, the fluorescence-activated cell sorting (FACS) Caliber flow cytometer (Becton Dickinson, San Jose, CA, USA) was used to assess EPC mobilization [18]. A volume of 100 $\mu \mathrm{L}$ peripheral blood was incubated with Fluorescein isothiocyanate (FITC) anti-mouse Sca-1 (eBioscience, San Diego, CA, USA), and phycoerythrin (PE) anti-mouse Flk-1 (VEGFR-2, eBioscience) antibodies. Isotypeidentical antibodies served as controls (Becton Dickinson, Franklin Lakes, NJ, USA). After incubated for 30 minutes, cells were lysed (PharmLyse; BD Pharmingen), washed with phosphate-buffered saline (PBS), and fixed in $2 \%$ paraformaldehyde before analysis. Each analysis included 100,000 events. Circulating EPCs were considered to be from the mononuclear cell population and were gated with double positive for Sca-1 and Flk-1.

\section{Bone marrow transplantation model}

Recipient wild-type mice at 8 weeks of age were lethally irradiated with a total dose of 9.0 Gy $[18,20]$. eGFP transgenic mice (FVB background) that ubiquitously expressed enhanced GFP (Level Biotechnology Inc., Taipei, Taiwan) were used as the donors. After being irradiated, a recipient mouse received unfractionated bone marrow cells $\left(5 \times 10^{6}\right)$ from an eGFP mouse by a tail vein injection. Two months after the bone marrow transplantation, the chimeric mice were induced diabetes by daily intravenous injections of STZ as previously described. All mice received a hindlimb ischemic surgery $(n=6$ in each group). Repopulation by eGFP-positive bone marrow cells was $95 \%$, as measured by flow cytometry. Two weeks after the induction of hindlimb ischemic surgery in the bone marrow-reconstituted and STZ-induced diabetic mice, tissues were harvested for confocal immunofluorescent and histological analysis. Bone marrow-derived EPCs were stained with antibodies directed against eGFP (Chemicon) and CD31 (BD PharMingen). EPC density was estimated by counting $\mathrm{eGFP}^{+} \mathrm{CD} 31^{+}$double-positive cells (yellow color) under high power field $(\mathrm{HPF}, \times 100)$ in at least 6 different cross-sections from different animals.

\section{EPC transplantation in nude mice}

Athymic nude mice at 6-8 weeks old were purchased from the National Laboratory Animal Center, Taiwan. Nude mice were then randomly assigned to 5 treatment groups ( $n=6$ in each group) for intramuscular injection of normal saline, healthy EPC, EPC treated with high glucose for 4 days (EPC-HG), EPC treated with high glucose and IFR for 30 mins (24 hours following by treatment of EPCs in high-glucose conditions for 4 days) (EPC-HG + IFR), and EPC treated with high glucose and transfected with eNOS siRNA (Santa CruzBiotechnology Inc., CA, USA) and IFR for 30 mins (EPC-HG + eNOS siRNA + IFR). EPCs were labeled with fluorescent carbocyanine 1,1'-dioctadecyl-1 to $3,3,3^{\prime}, 3^{\prime}-$ tetramethylindocarbocyanine perchlorate (DiI) dye (Molecular Probes) [21]. Intramuscular injection was performed 24 hours following unilateral hindlimb ischemia srugery. A total volume of $200 \mu \mathrm{l}$ normal saline or $5 \times 10^{6}$ EPC were injected at 6 sites into the ischemic hind limb distal to the arterial occlusion site. Three ventral injections were placed in the upper limb in proximity to the adductor and semimembranosus muscles. The remaining 3 injections were administered to the ventral lower limb involving the gastrocnemius and flexor digitorum muscles. In order to achieve maximal experimental uniformity, transplanted EPCs were derived from the same donors and used in parallel experiments.

\section{Human EPC isolation and cultivation}

Peripheral blood samples $(20 \mathrm{ml})$ were obtained from healthy young adult volunteers, and total mononuclear cells (MNCs) were isolated by density gradient centrifugation with Histopaque-1077 (1.077 g/ml, Sigma, St. Louis, MO, USA) [20]. Briefly, MNCs $\left(5 \times 10^{6}\right)$ were plated in $2 \mathrm{ml}$ endothelial growth medium (EGM-2 MV Cambrex, East Rutherford, NJ, USA) with supplements on fibronectin-coated 6-well plates. After 4 days of culturing, the medium was changed and non-adherent cells were removed; attached early EPCs appeared to be elongated with spindle shapes. A certain number of early EPCs were allowed to grow into ECFCs (endothelial colonies forming cells), which emerged 2-4 weeks after the start of the MNC culture. The ECFCs exhibited a cobblestone morphology and monolayer growth pattern typical of mature endothelial cells at confluence [22]. ECFCs were collected and used for all the assays in this study.

\section{ECFC characterization}

The ECFCs were characterized as adherent cells that were positive for endothelial cell and haematopoietic stem cell surface makers, as previously described [20]. The ECFCs were characterized by immunofluorescence staining for the expression of VE-cadherin, platelet/ 
endothelial cell adhesion molecule-1 (PECAM-1, CD-31), and CD34, KDR, AC133 and eNOS (Santa Cruz). The fluorescent images were recorded under a laser scanning confocal microscope.

\section{Measurement of reactive oxygen species (ROS) production and measurement of nitrate levels}

The effect of IFR therapy on ROS production in ECFCs was determined by a fluorometric assay using 2',7'dichlorofluorescein diacetate (DCFH-DA, Molecular Probes) as a probe for the presence of $\mathrm{H}_{2} \mathrm{O}_{2} \cdot{ }^{21}$ ECFCs $\left(10^{4}\right.$ cells/well $)$ in 96-well plates was incubated in high glucose medium for 4 days. After treatment of IFR radiation, cells were incubated with $20 \mu \mathrm{mol} / \mathrm{L}$ DCFH-DA for 45 minutes. The conditioned medium was measured for nitrate level by Griess reagent [1\% sulfanilamide and $0.1 \% N$-(1-naphthyl) ethylenediamine in $2 \%$ phosphoric acid]. The fluorescence intensity (relative fluorescence units) was assessed at 485-nm excitation and 530-nm emission using a fluorescence microplate reader.

\section{EPC senescence assay}

Cellular aging was determined with a Senescence Cell Staining kit (Sigma). Briefly, after washing with PBS, ECFCs were fixed for 6 minutes in $2 \%$ formaldehyde and $0.2 \%$ glutaraldehyde in PBS, and then incubated for 12 hours at $37^{\circ} \mathrm{C}$ without $\mathrm{CO}_{2}$ in fresh $\mathrm{X}$-gal staining solution $(1 \mathrm{mg} / \mathrm{ml} \mathrm{X}$-gal, $5 \mathrm{mM}$ potassium ferricyanide, and $2 \mathrm{mM} \mathrm{MgCl}$; pH6). After staining, green-stained cells and total cells were counted and the percentage of $\beta$-galactosidase-positive cells was calculated [20].

\section{EPC migration assay}

The migratory function of ECFCs was evaluated by a modified Boyden chamber assay (Transwell, Costar) [20]. Briefly, isolated ECFCs were detached as described above with trypsin/EDTA, and then $4 \times 10^{4}$ ECFCs were placed in the upper chambers of 24-well transwell plates with polycarbonate membranes $(8-\mu \mathrm{m}$ pores) that contained serum-free endothelial growth medium. VEGF (50 $\mathrm{ng} / \mathrm{ml}$ ) was added to medium placed in the lower chambers. After incubation for 24 hours, the membrane was washed briefly with PBS and fixed with $4 \%$ paraformaldehyde. The upper membrane side was wiped gently with a cotton ball. The membrane was stained using hematoxylin solution and carefully removed. The extent of migration of ECFCs was evaluated by counting the migrated cells in 6 random high-power $(\times 100)$ microscopic fields.

\section{Western blotting analysis}

ECFCs were lysed in buffer $(62.5 \mathrm{mM}$ Tris- $\mathrm{HCl}, 2 \%$ SDS, 10\% glycerol, $0.5 \mathrm{mM}$ PMSF, $2 \mu \mathrm{g} / \mathrm{ml}$ aprotinin, pepstatin and leupeptin), and the protein lysates were subjected to SDS-PAGE, followed by electroblotting onto a PVDF membrane [12]. Membranes were probed with monoclonal antibodies against phosphorylated endothelial NO synthase (p-eNOS), eNOS, Akt, p-Akt, VEGF, p-ERK, p-38 MAPK, HO-1 (Cell Signaling) and $\beta$-actin (Sigma). Bands were visualized by chemiluminescence detection reagents. Densitometric analysis used ImageQuant (Promega) software.

\section{Statistical analysis}

Results are given as means \pm standard errors of the mean (SEM). Statistical analysis was done by unpaired Student's $t$ test or analysis of variance, followed by Scheffe's multiple-comparison post hoc test. Data were analyzed using SPSS software (version 14; SPSS, Chicago, IL). A $\mathrm{p}$ value of $<0.05$ was considered statistically significant.

\section{Results}

\section{IFR therapy promotes blood flow recovery in diabetic} mice

Local IFR therapy was given in STZ-induced diabetic mice for 30 min twice per day for 2 weeks after the surgery, and wild-type and diabetic control mice were placed on a heating plate at $34^{\circ} \mathrm{C}$ for 30 min twice daily to avoid the thermal effect in this study. As shown in Figure 1A, the STZ-induced diabetic mice without IFR therapy showed delayed blood flow recovery after ischemia surgery compared with that in wild-type mice, as determined by Laser Doppler imaging. Meanwhile, the repeated IFR therapy significantly improved blood flow recovery by $48 \%$ in STZ-induced diabetic mice ( $n=6$ per group). However, the benefit of local IFR radiation was significantly abolished after treatment with the eNOS inhibitor $\mathrm{N}^{\mathrm{G}}$-nitro-L-arginine methyl ester (L-NAME, $1 \mathrm{mg} / \mathrm{ml}$ in drinking water). Consistent with the measurements by Laser Doppler imaging, anti-CD31 immunostaining revealed that repeated FIR radiation increased the number of detectable capillaries in the ischemic muscle in STZinduced diabetic mice (control versus IFR: $38.8 \pm 1.8$ versus $48.7 \pm 2.4 / \mathrm{HPF}, \mathrm{p}=0.008$ ) (Figure $1 \mathrm{~B}$ ). However, administration of L-NAME abolished the benefit of IFR radiation (detectable capillaries, IFR versus IFR + L-NAME: $48.7 \pm 2.4$ versus $34.8 \pm 1.7 / \mathrm{HPF}, \mathrm{p}=0.001)$.

\section{Effects of IFR radiation on oxidative stress in ischemic limbs, EPC mobilization and homing process}

To further evaluate the effect of IFR therapy on oxidative stress on ischemic muscles, immunostaining against nitrotyrosine was performed. As shown on Figure 2A, assessed by nitrotyrosine staining, a significant reduction of oxidative stress levels in ischemic muscles was noted in diabetic mice that received IFR therapy. 


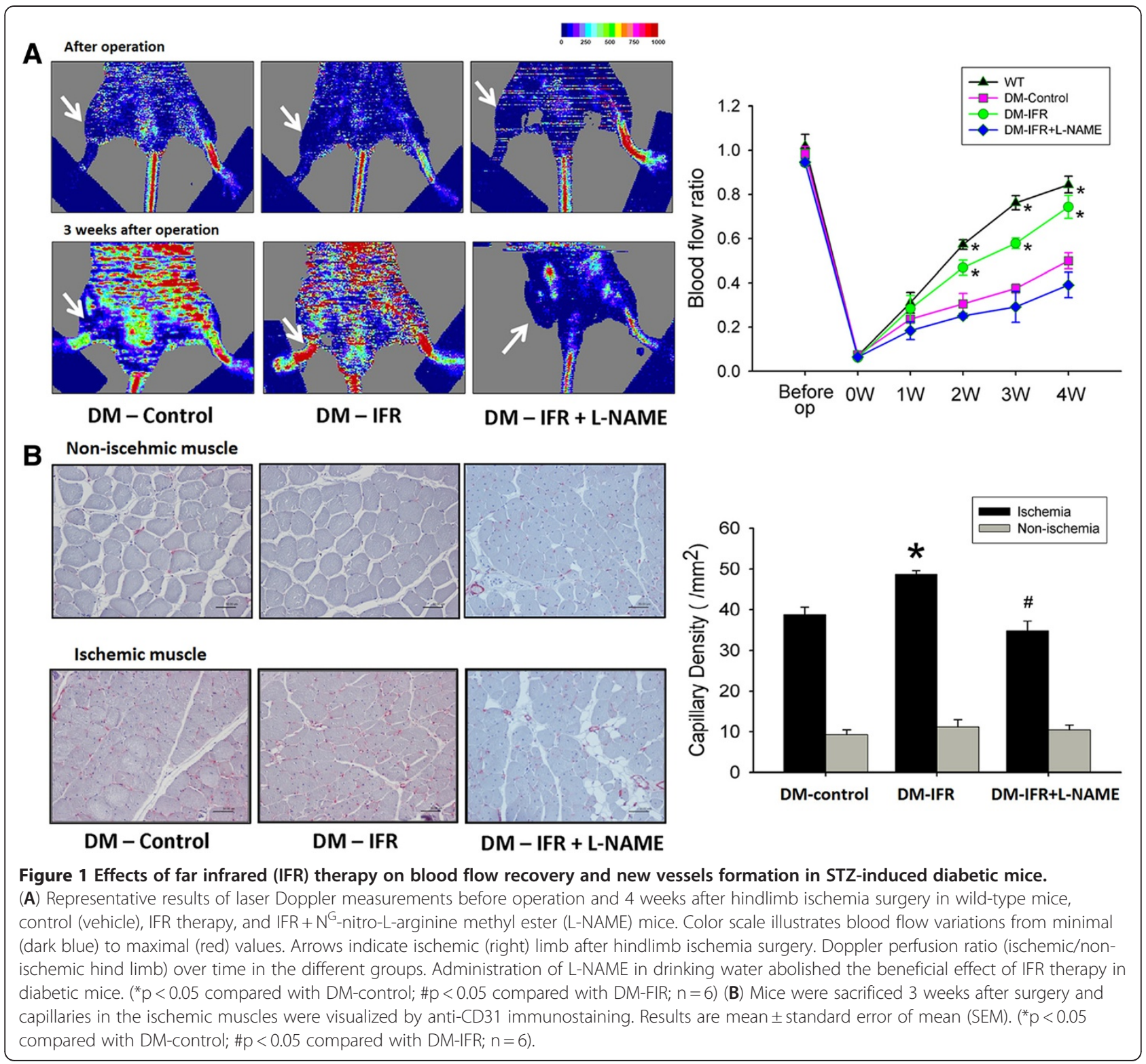

To investigate the effects of repeated IFR radiation on EPC mobilization in response to tissue ischemia, levels of Sca- $1^{+} / \mathrm{Flk}_{-} 1^{+}$cells in peripheral blood were determined by flow cytometry in STZ-induced diabetic mice ( $n=6$ per group). EPCs mobilization was enhanced by tissue ischemia in wild-type mice (baseline versus 2 days after operation: $0.60 \pm 0.22$ versus $1.34 \pm 0.11 \%$, $\mathrm{p}=0.016$ ). However, impaired mobilization of EPCs in peripheral blood after hindlimb ischemia was noted in STZ-induced diabetic mice (baseline vs. 2 days after operation: $0.45 \pm 0.07$ vs. $0.56 \pm 0.09 \%, p=0.374)$. As shown in Figure $1 \mathrm{C}$, administration of local IFR radiation did not increase EPC mobilization in STZinduced diabetic mice (baseline versus 2 days after operation: $0.36 \pm 0.12$ versus $0.61 \pm 0.10 \%, \quad \mathrm{p}=0.140$ ) after hindlimb ischemia surgery.

To test the effect of repeated IFR radiation on bone marrow-derived EPC homing and differentiation to endothelial cells, STZ-induced diabetes was created in FVB mice that received eGFP mouse bone marrow cells. By immunofluorescence staining, STZ-induced diabetic mice in the IFR group had more $\mathrm{GFP}^{+} / \mathrm{CD} 31^{+}$doublepositive cells in ischemic muscle than those in control group (control versus IFR: $11.2 \pm 1.1$ versus $18.8 \pm 2.0$ / $\mathrm{HPF}, \mathrm{p}=0.007, \mathrm{n}=6$; Figure 2 ). In addition, administration of the eNOS inhibitor significantly diminished the effect of IFR radiation on EPC homing. These results suggested that repeated IFR therapy did not promote 


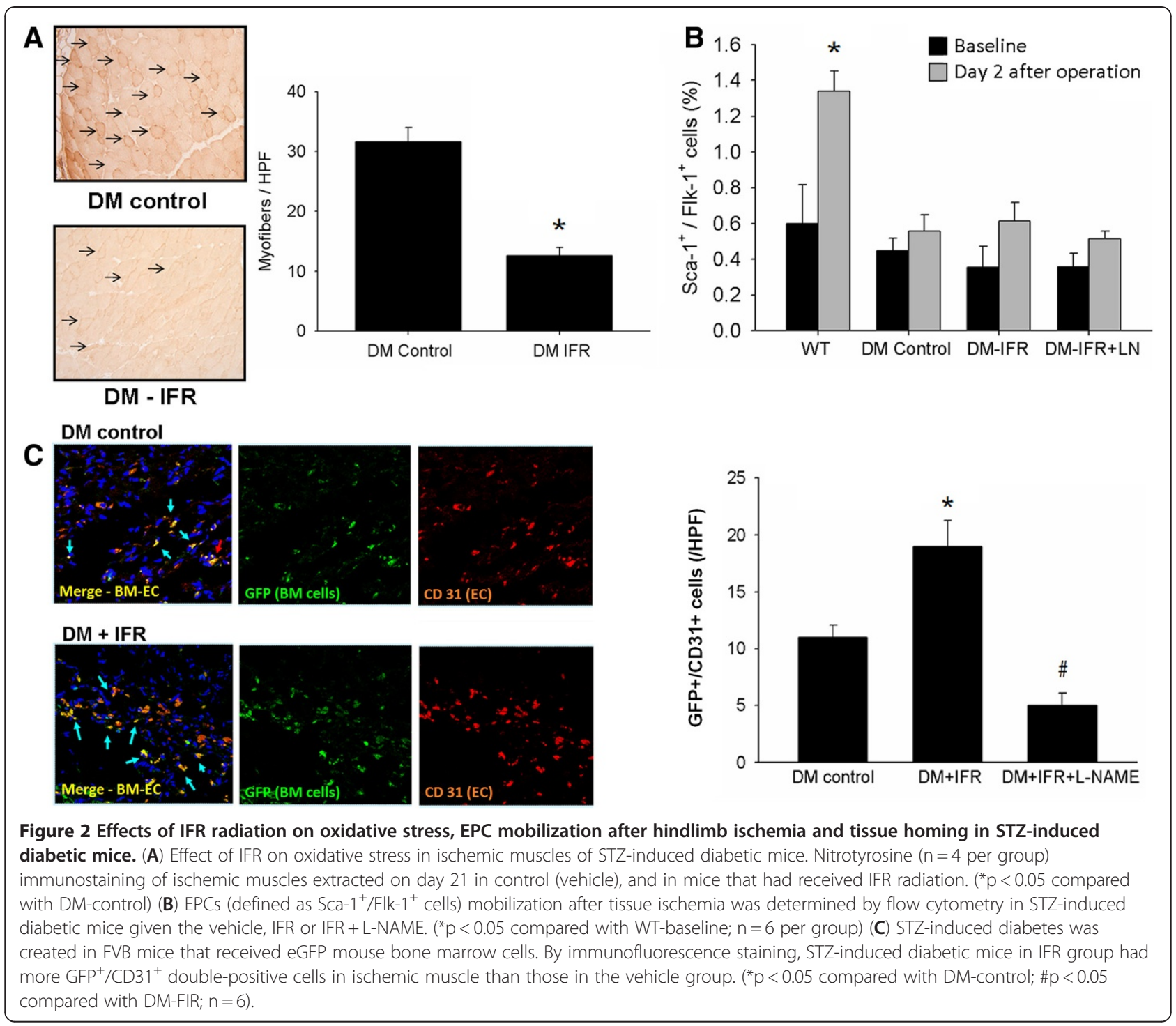

EPC mobilization after tissue ischemia but may increase circulating EPCs' homing to ischemic tissue.

\section{Characterization of Human ECFCs}

ECFCs were isolated from peripheral blood MNCs of healthy young adult volunteers as previously described [22]. The peripheral blood MNCs that initially seeded on fibronectin-coated wells were in round shape (Figure 3A). After the medium was changed on day 4, attached MNCs appeared to be elongated with a spindle shape (Figure 3B). ECFCs with a cobblestone-like morphology similar to mature endothelial cells were grown to confluence (Figure 3C). ECFCs characterization was performed by immunohistochemical staining, and most of the cells expressed mature endothelial markers, VE-cadherin, PECAM-1 (CD31), CD34, KDR, AC133 and eNOS (Figure 3), which are considered as critical markers of late EPCs.
IFR decreases reactive oxidative stress and enhances EPC proliferation, NO production in high glucose conditions High glucose markedly increased $\mathrm{H}_{2} \mathrm{O}_{2}$ production determined by the relative DCFH-DA fluorescent intensity, and administration of IFR radiation (10-60 mins) significantly suppressed the high glucose-induced ROS index in cultures of ECFCs (Figure 4A).

As shown in Figure 4B, incubation with high glucose medium for 4 days significantly reduced EPC proliferation (control versus high glucose, $100 \pm 2$ versus $88 \pm 5$, $\mathrm{p}=0.026$ ). However, administration of IFR radiation for $40 \mathrm{~min}$ following by treatment of EPCs in high-glucose conditions significantly reversed the reduction in EPC proliferation in response to high glucose (high glucose versus high glucose + IFR 40 mins, $88 \pm 5$ versus $109 \pm 2$, $\mathrm{p}=0.006)$.

High glucose impairs eNOS activation and reduces NO bioavailability in cultured EPCs [7]. We therefore 

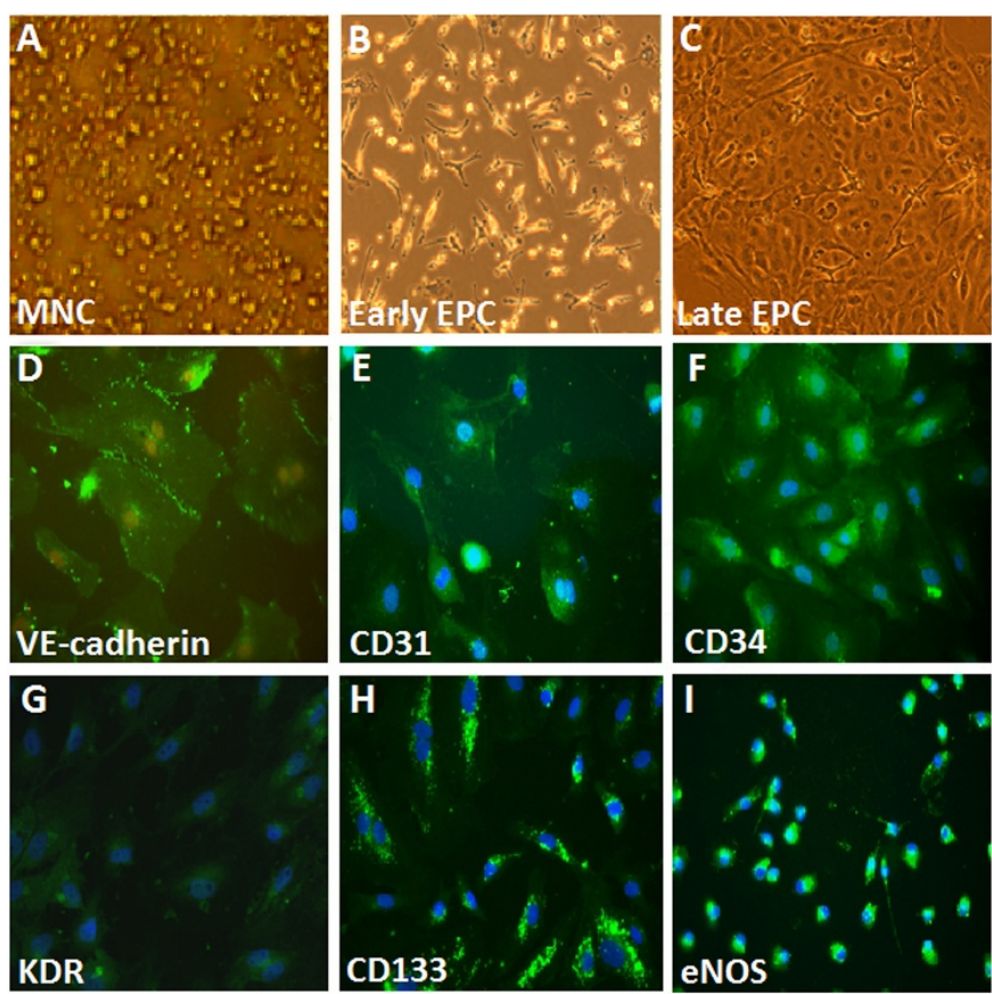

Figure 3 Morphology and characterization of human endothelial progenitor cells (EPCs) from peripheral blood. (A) Peripheral blood mononuclear cells (MNCs) were plated on a fibronectin-coated culture dish on the first day. (B) Four days after plating, adherent early EPCs with a spindle shape were shown. (C) Three weeks after plating, ECFCs with a cobblestone-like morphology were selected, reseeded, and grown to confluence. (D-I) ECFC characterization was performed by immunohistochemical staining. Most of the EPC expressed endothelial and

hematopoietic stem cell markers, VE-cadherin, PECAM-1 (CD31), CD34, KDR, AC133, and eNOS, which are considered critical markers of EPCs. Cells were counterstained with 4',6-diamidino-2-phenylindole (DAPI) for the nuclei (blue).

tested the effects of IFR radiation on high glucosetreated EPCs to determine whether IFR could recover impaired NO production in EPCs. After 4 days of incubation in $25 \mathrm{mM}$ high glucose medium, the NO production in cultured medium was significantly decreased (Figure 4C). However, administration of IFR radiation for 30 mins following by treatment of EPCs in highglucose conditions can significantly upregulate high glucose-impaired No production.

\section{IFR radiation upregulates phosphorylation of eNOS, and} enhances VEGF in high glucose conditions

We investigated the effects of IFR radiation on high glucose-treated EPCs to determine whether IFR therapy could recover impaired eNOS activation in EPCs. Administration of IFR radiation on cultured ECFCs for 10, 30, and $40 \mathrm{~min}$ followed by treatment of EPCs in high glucose conditions significantly upregulated high glucose-impaired eNOS production and eNOS activity (p-eNOS/total eNOS). Treatment of IFR therapy for 30, 40 and 60 min also upregulated Akt activation (p-Akt/ total Akt). In addition, administration IFR radiation also promoted VEGF production, p-ERK, and p-38 MAPK in high glucose conditions, but not HO-1 (Figure 5). These findings suggested that treatment with IFR radiation may enhance activities of eNOS, Akt, p-ERK, p-38 MAPK and VEGF production in EPCs in response to high glucose stimulation.

\section{IFR improves high glucose-suppressed EPC functions and senescence in vitro}

To investigate the effects of IFR radiation on EPCs, we used both scratch test and modified Boyden chamber assay to assess the migratory function of EPCs in high glucose conditions. Compared with the control group, incubation of EPCs with high glucose (4 days) significantly decreased EPC migration $(100 \pm 9$ versus $63 \pm 11 / \mathrm{HPF}, \mathrm{p}=0.031$ ). However, treatment with IFR radiation for 30 minutes significantly recovered high glucose-suppressed late EPC migratory function (high glucose versus high glucose + IFR, $63 \pm 11$ versus $174 \pm 10$ cells $/ H P F, p=0.005$; Figure $6 A$ ). Similar findings were observed by modified Boyden chamber assay (Figure 6B).

After 4 days of culturing, the capacity for tube formation of EPCs on ECMatrix gel was significantly reduced 


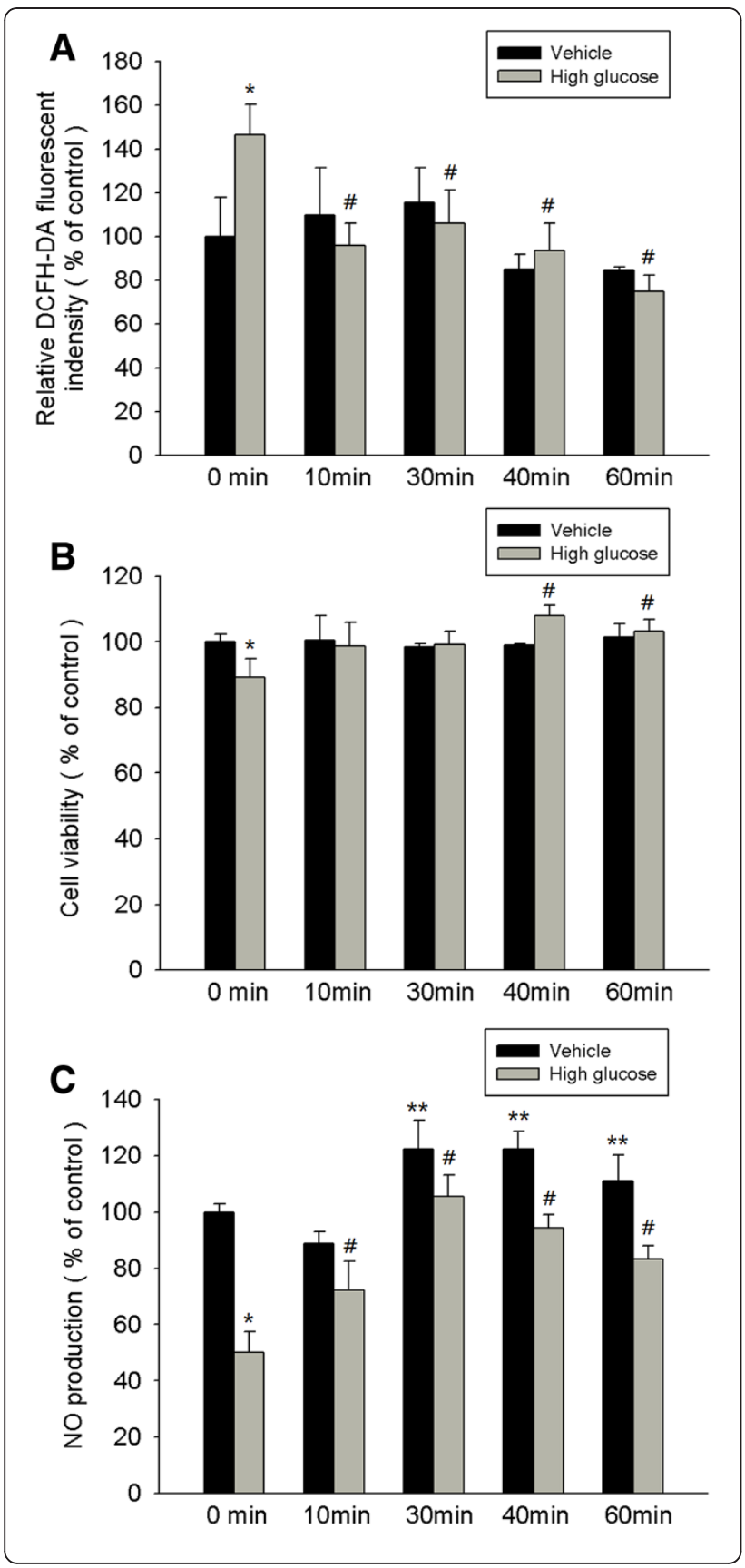

in the presence of high glucose compared with the control group, whereas treatment with IFR radiation for 30 mins followed by treatment of EPCs in high-glucose conditions ameliorated this high glucose suppressedtube formation by EPCs $(100 \pm 15$ vs. $195 \pm 3$ cells/HPF, $\mathrm{p}=0.003$; Figure 6C).

Compared with the control group, incubation of EPCs with high glucose significantly increased the percentage of senescence-associated ß-galactosidase-positive EPCs ( $100 \pm 6$ versus $119 \pm 5 \%, \mathrm{p}=0.032)$. Administration of IFR radiation (30 mins) followed by treatment of EPCs
Figure 4 IFR therapy decreased reactive oxidative stress, recovered EPC proliferation, and increased $\mathrm{NO}$ production in high-glucose conditions. (A) High glucose markedly increased $\mathrm{H}_{2} \mathrm{O}_{2}$ production determined by the relative DCFH-DA fluorescent intensity, and the administration of IFR therapy suppressed high glucose-induced reactive oxidative stress (ROS) index in EPCs culture. $\left({ }^{*} p<0.05\right.$ compared with control - 0 min; \#p $<0.05$ compared with high glucose - 0 min) (B) The effect of IFR radiation on EPCS proliferation was analyzed by MTT assay. ( ${ }^{*} p<0.05$ compared with control - 0 min; \#p < 0.05 compared with high glucose - 0 min) (C)

Nitrate production (as NO content) in culture medium was measured by Griess reagent. High glucose-suppressed NO production in cultured late EPC s. After 4 days of incubation, IFR radiation increased NO production with or with high glucose conditions. ( ${ }^{*} p<0.05$ compared with control - 0 min; \#p $<0.05$ compared with high glucose -0 min; ${ }^{* *} p<0.05$ compared with control - 0 min; $n=4$ for each experiment).

in high-glucose medium significantly attenuated the percentage of senescence-associated $ß$-galactosidase-positive EPCs (high glucose versus high glucose + IFR, $119 \pm 5$ versus $87 \pm 9 \%, p=0.013$; Figure $6 D$ ).

\section{EPC treated IFR radiation transplantation improves hindlimb perfusion}

In the animals receiving normal saline, blood flow remained constant throughout the study, around $42 \%$ of that measured in the non-ischemic limb (42 $\pm 8 \%$, four weeks after operation). In contrast, the mice transplanted with EPC and EPC received IFR therapy (30 mins) in high glucose medium (EPC-HG + IFR), but not EPC in high glucose medium without IFR therapy (EPCHG) and EPC treated with high glucose and IFR and eNOS siRNA (EPC-HG + eNOS siRNA + IFR) already showed a significant improvement in blood flow by three weeks after EPCs implantation (both $\mathrm{p}<0.05$; Figure 7 ). Consistent with the measurements by Laser Doppler imaging, anti-CD31 immunostaining revealed that transplantation of EPC and EPC treated with IFR in high glucose conditions significantly increased the number of detectable capillaries in the ischemic muscle than mice received normal saline (both $\mathrm{p}<0.05$ compared to normal saline). However, treatment with eNOS siRNA abolished the benefit of IFR radiation in high glucose medium (IFR versus IFR + eNOS siRNA: $38.7 \pm 4.7$ versus $21.3 \pm 3.7 / \mathrm{HPF}, \mathrm{p}=0.015, \mathrm{n}=6$ per group).

\section{Discussion}

This study first to show a favorable effect of IFR radiation on ischemia-induced neovascularization in diabetic mice. Repeated IFR radiation enhanced blood flow recovery and new vessel formation in ischemic hindlimbs, and the beneficial effect may be derived from enhancement of EPC homing process by reduction of oxidative stress in ischemic tissue rather than promotion of EPC 

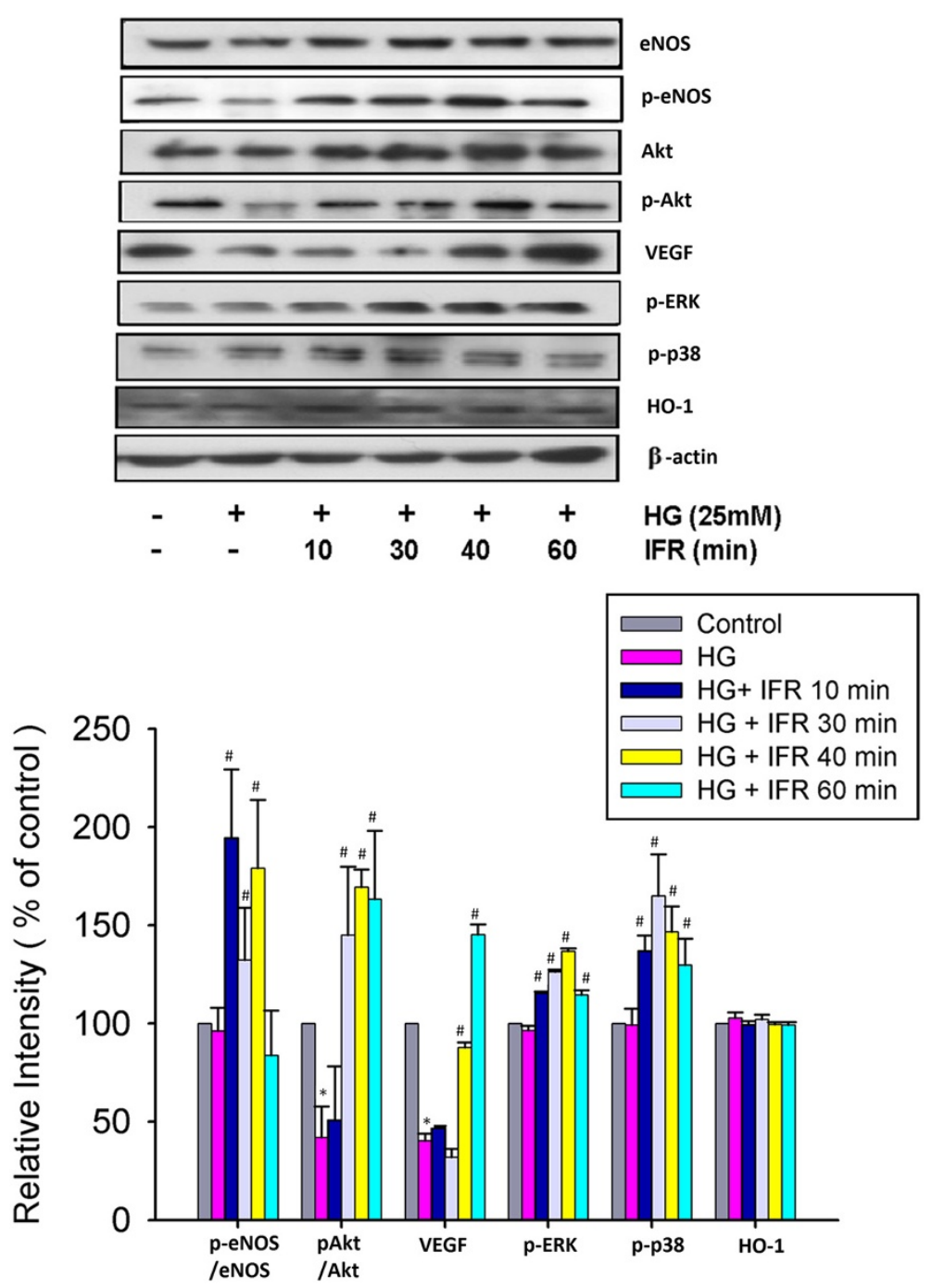

Figure 5 Effects of IFR radiation on eNOS, Akt, p-ERK, p-38 MAPK, VEGF and HO-1 production in cultured EPCs. Administration of IFR radiation on cultured ECFCs for 10, 30, and 40 min followed by treatment of EPCs in high-glucose conditions significantly upregulated high glucose-impaired eNOS production and eNOS activity (p-eNOS/total eNOS). Treatment of IFR therapy for 30, 40 and 60 min also upregulated Akt activation (p-Akt/total Akt). In addition, administration IFR radiation also promoted VEGF production, p-ERK, and p-38 MAPK in high glucose conditions, but not HO-1. Each bar graph shows the summarized data from four separate experiments by densitometry after normalization. Data are means $\pm S E M ; n=4$ in each experiment. ( ${ }^{*} p<0.05$ compared with control; ${ }^{*} p<0.05$ compared with HG group.).

mobilization. In addition, direct IFR radiation could ameliorate high glucose-induced oxidative stress, attenuate cellular senescence and improve EPC functions. Mice received EPC treated with IFR radiation showed a significant improvement in blood flow recovery after tissue ischemia in comparison to those received normal saline. Given the evidence mentioned above, our findings indicated the direct beneficial effects of IFR radiation on blood flow recovery after tissue ischemia, and IFR radiation counteracts the detrimental effect of a diabetic environment on improvement of EPC functions, which may provide some novel rationales for its potential clinical impact on vascular protection.
Improved neovascularization in response to tissue ischemia is an important therapeutic strategy to reduce organ damage. Convincing evidence suggests that neovascularization in adults is not solely the result of the proliferation of endothelial cells (angiogenesis) but also involves circulating EPCs in the process of vasculogenesis [7]. These circulating EPCs are derived from bone marrow and are mobilized endogenously, triggered by tissue ischemia, or exogenously by cytokine stimulation, such as VEGF and stromal cell-derived factor-1 (SDF-1) [23].

However, patients with diabetes or cardiovascular risk factors were shown to have decreased numbers and 
A

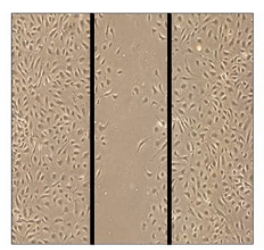

HG 30 min

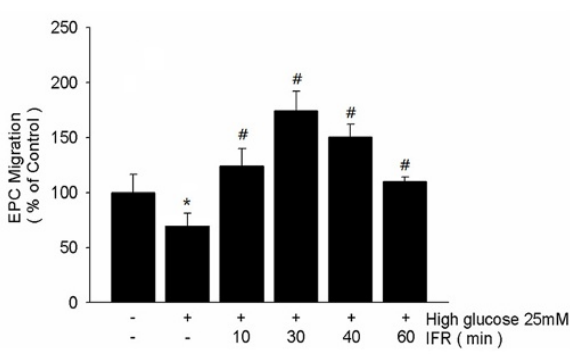

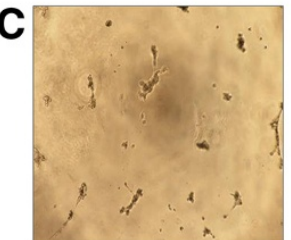

HG 30 min

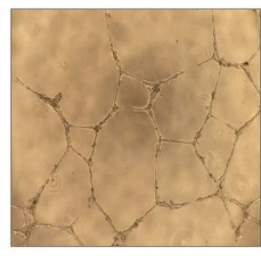

IFR $30 \mathrm{~min}$

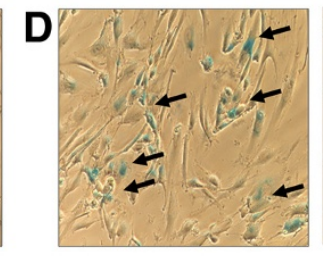

HG $30 \mathrm{~min}$

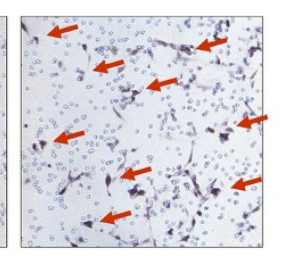

IFR $30 \mathrm{~min}$

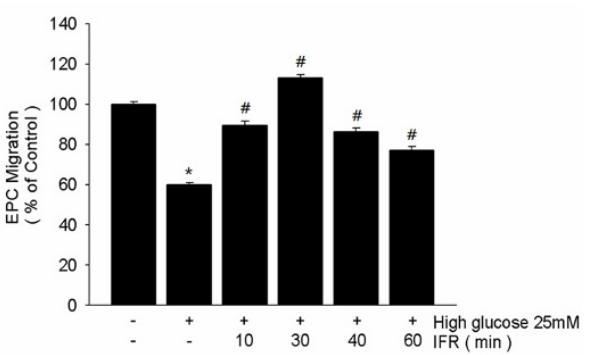

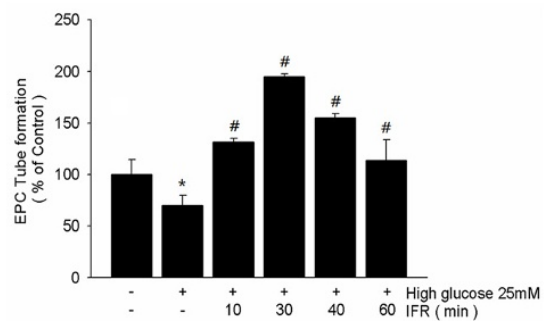

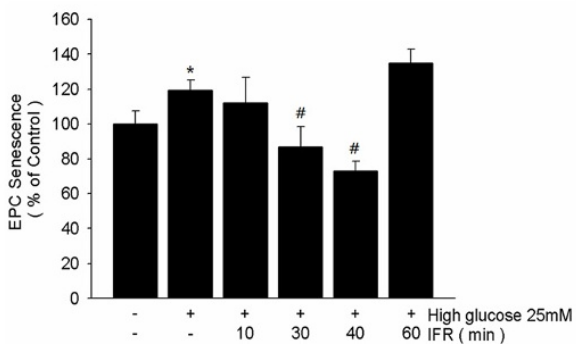

Figure 6 Effects of IFR therapy on EPC migration, tube formation, and senescence in vitro. (A,B) Scratch test and modified Boyden chamber assay were used to assess the migratory function of ECFCs in high glucose conditions. Boyden chamber assay using VEGF as a chemoattracting factor was used to evaluate the effects of IFR radiation on EPC migration. (C) An in vitro angiogenesis assay for late ECFCs used ECMatrix gel. Representative photos for in vitro angiogenesis are shown. Cells were stained with crystal violet, and the averages of the total area of complete tubes formed by cells were compared by using computer software. (D) To determine the onset of cellular aging, acidic Bgalactosidase was used as a biochemical marker for acidification, typical for ECFCs senescence. Data are means \pm SEM; $n=4$ in each experiment.

function of circulating EPCs $[8,24,25]$. Recent studies indicated that advanced glycation end products (AGEs) promoted EPC apoptosis [26], and long-term exposure to high glucose may enhance cellular senescence and reduce cell numbers and functional competencies of EPCs via NO-related mechanisms [10]. Mobilization and differentiation of EPCs are modified by NO, and bone marrow-expressed eNOS is essential for the mobilization of stem and progenitor cells [27]. In addition, endogenous NOS inhibitors, such as asymmetric dimethylarginine (ADMA), were shown to suppress EPC differentiation and function, and contributed to impaired endothelial function [28]. These findings provide a rationale for potential therapeutic targets for hyperglycemia- suppressed EPC functions, and diabetes-related vascular complications.

Diabetic patients frequently suffer from micro- or macrovascular abnormalities, including retinopathy, nephropathy, neuropathy and accelerated atherosclerosis. It is evident that decreased bioavailability of $\mathrm{NO}$ produced from eNOS plays a crucial role in the development and progression of atherosclerosis. Under various pathological conditions such as type 2 diabetes eNOS may become dysfunctional or its expression may be decreased. Endothelial dysfunction is associated with childhood obesity and is closely linked to the amount and function of EPCs, and a combined after-school exercise program was shown to increase circulating EPC 


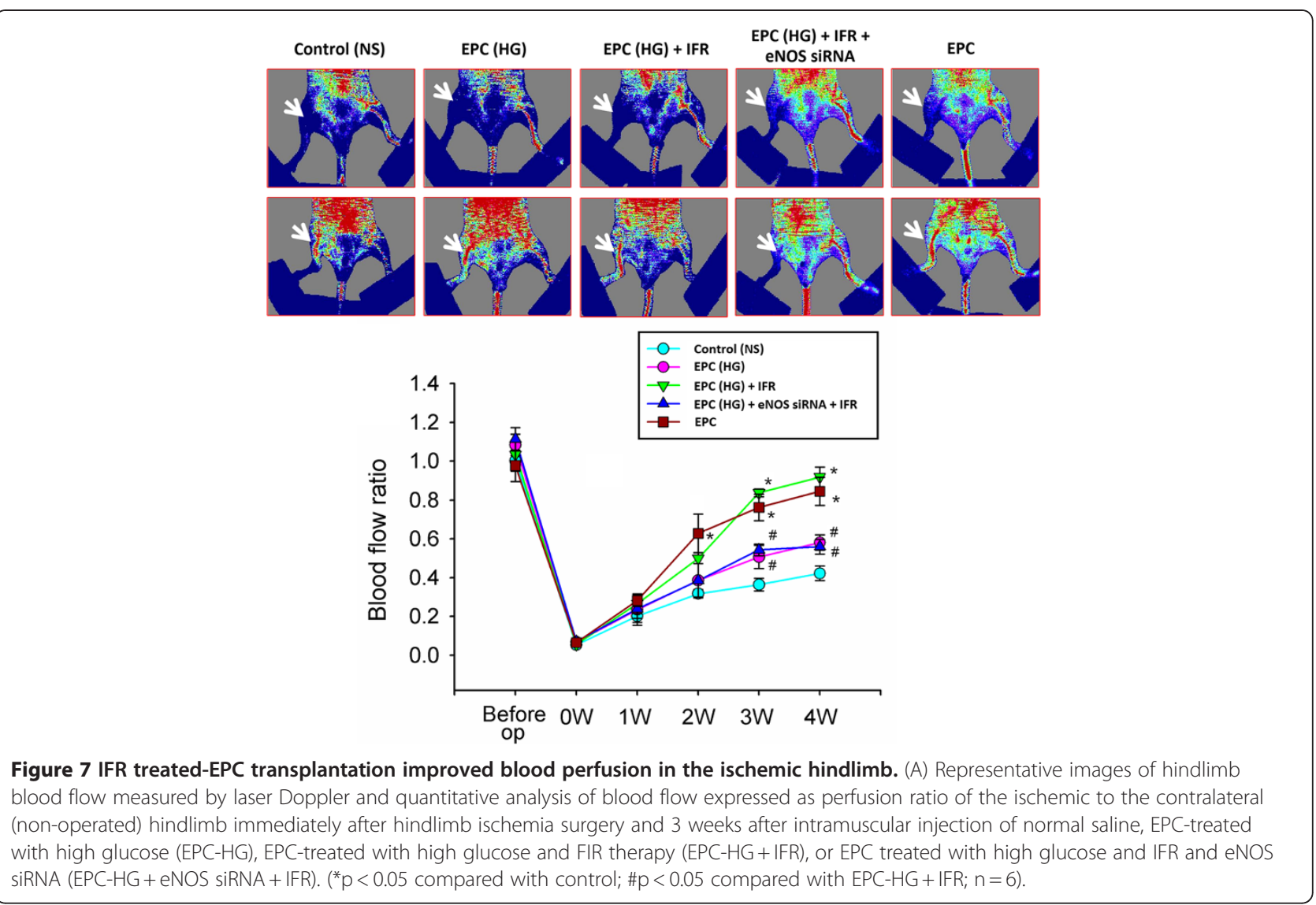

levels by enhancement of NO bioavailability [29]. Moreover, enhancement of oxidative stress by tissue ischemia may downregulate NO bioavailability because free radicals can directly inactivate NO [30]. Inadequate angiogenic response to ischemia in the ischemic limbs or myocardium of diabetic patients could result in poor collateral formation and severe organ damage.

IFR radiation is an invisible electromagnetic wave with a characteristic wavelength between 5.6 and $1000 \mu \mathrm{m}$ that can be perceived as heat by thermo-receptors in skin [11,12]. The technology of IFR has been applied widely in a variety of fields. The thermal effect of IFR results in vasodilation and increasing tissue blood flow. Local IFR therapy may allow multiple energy transfer as deep as 2 to $3 \mathrm{~cm}$ into subcutaneous tissue without irritating or overheating the skin like unfiltered heat radiation [31]. The skin temperature steadily increased to a plateau at approximately 38 to $39^{\circ} \mathrm{C}$ during the treatment of FIR for 30 to $60 \mathrm{~min}$ as long as the distance between the ceramic plate and the skin was $>20 \mathrm{~cm}$ [16]. Therefore, infrared therapy can be free of the disadvantages or adverse effects of thermal therapy. In this study, wild-type and diabetic control mice were placed on a heating plate at $34^{\circ} \mathrm{C}$ for $30 \mathrm{~min}$ twice daily to avoid the thermal effect between groups. The rectal temperature was also assessed in these mice, and showed no temperature elevation in mice received IFR therapy compared to those without IFR radiation.

In addition to the thermal effect, increasing evidence suggests nonthermal effects of IFR therapy exert beneficial effects in the cardiovascular system through NOrelated pathway [31,32]. In animal studies, Akasaki and colleagues demonstrated that repeated IFR therapy could upregulate eNOS expression and augment angiogenesis in an apolipoprotein E-deficient mouse model of unilateral hindlimb ischemia [16]. In human study, Imamura et al. showed that two weeks of repeated sauna therapy significantly improved vascular endothelial function, resulting in an increase of flow-mediated, endotheliumdependent dilation of the brachial artery from 4 to $5.8 \%$ in patients with coronary risk factors [14]. These findings suggested nonthermal effect of IFR may derive from upregulation of eNOS activity and enhancement of $\mathrm{NO}$ bioavailability. In the study, we demonstrated that repeated IFR therapy could activate eNOS and Akt, and upregulate the migration and tube formation capacities of ECFCs. Akt is downstream from PI 3-kinase and is capable of directly phosphorylating eNOS at Ser ${ }^{1179}$, resulting in its activation. Recent evidence also indicated that p-38 MAPK plays a key role in downregulating 
EPCs by hyperglycemia in diabetic patients [33]. EPCs exposed to IFR upregulated expression of p-38 MAPK and p-ERK. These findings are in line with recent study showing that IFR radiation significantly promoted angiogenesis by a MAP kinase dependent pathway on mature endothelial cells. Mice received EPC treated with IFR radiation in high glucose conditions showed a significant improvement in blood flow recovery after tissue ischemia compared with those received normal saline. These beneficial effects may provide some novel rationale for the vascular protective properties of IFR therapy in diabetic patients with critical limb ischemia or clinical implication to treat dysfunctional EPCs before cell therapy.

\section{Conclusions}

This study provides the first evidence that the administration of IFR therapy promoted collateral flow recovery and new vessel formation in STZ-induced diabetic mice. These beneficial effects may derive from enhancement of EPC functions and homing process, which provide some novel rationale for the vascular protective properties of IFR therapy in diabetic patients with critical limb ischemia or further clinical implication in cell therapy.

\begin{abstract}
Abbreviations
EPCs, Endothelial progenitor cells; NO, Nitric oxide; DM, Diabetes mellitus; IFR, Far infrared; STZ, Streptozotocin; FACS, Fluorescence-activated cell sorting; FITC, Fluorescein isothiocyanate; PE, Phycoerythrin; PBS, Phosphatebuffered saline; MNCs, Mononuclear cells; ECFCs, Endothelial colonies forming cells; PECAM-1, Platelet/endothelial cell adhesion molecule-1; ROS, Reactive oxygen species; DCFH-DA, 2',7'-dichlorofluorescein diacetate; p-eNOS, Phosphorylated endothelial NO synthase; L-NAME, $N^{G}$-nitro-Larginine methyl ester; SDF-1, Stromal cell-derived factor-1; AGEs, Advanced glycation end products; ADMA, Asymmetric dimethylarginine.
\end{abstract}

\section{Competing interests}

The authors declare that they have no competing interests.

\section{Authors' contributions}

Po-Hsun Huang conducted the experiments and contributed to the study implementation, statistical analysis, interpretation, and the preparation of the manuscript. Jaw-Wen Chen contributed to the study conception and design, and the preparation of the manuscript. Chih-Pei Lin, Ying-Hwa Chen, ChaoHung Wang, and Hsin-Bang Leu helped to conduct the experiments and contributed to the study conception and design, implementation, and interpretation. Jaw-Wen Chen and Shing-Jong Lin supervised the study conduction and contributed to the study conception and design, implementation, statistical interpretation, the preparation and finalization of the manuscript. All authors approved the final manuscript for publication.

\section{Acknowledgements}

This study was supported in part by research grants from the NSC 98-2314B-075-035, and UST-UCSD International Center of Excellence in Advanced Bio-engineering NSC-100-2911-I-009-101-A2 from the National Science Council; VGH-V98B1-003 and VGH-V100E2-002 from Taipei Veterans General Hospital. The funders had no role in study design, data collection, and analysis, decision to publish, or preparation of the manuscript.

\section{Author details}

${ }^{1}$ Division of Cardiology, Taipei Veterans General Hospital, Taipei, Taiwan. ${ }^{2}$ Department of Medical Research and Education, Taipei Veterans General Hospital, No. 201, Sec. 2, Shih-Pai Road, Taipei, Taiwan. ${ }^{3}$ Department of Pathology and Laboratory Medicine, Division of General Laboratory, Taipei Veterans General Hospital, Taipei, Taiwan. ${ }^{4}$ Healthcare and Management
Center, Taipei Veterans General Hospital, Taipei, Taiwan. ${ }^{5}$ Institute of Clinical Medicine, National Yang-Ming University, Taipei, Taiwan. ${ }^{6}$ Cardiovascular Research Center, National Yang-Ming University, Taipei, Taiwan. ${ }^{7}$ Institute and Department of Pharmacology, National Yang-Ming University, Taipei, Taiwan. ${ }^{8}$ Department of Biotechnology and Laboratory Science in Medicine and Institute of Biotechnology in Medicine, National Yang-Ming University, Taipei, Taiwan. ${ }^{9}$ Division of Cardiology, Chang Gung Memorial Hospital, Keelung, and Chang Gung University College of Medicine, Taoyuan, Taiwan.

Received: 3 July 2012 Accepted: 8 August 2012

Published: 15 August 2012

\section{References}

1. Folkman J: Seminars in Medicine of the Beth Israel Hospital, Boston. Clinical applications of research on angiogenesis. N Engl J Med 1995, 333(26):1757-1763.

2. Freedman S, Isner JM: Therapeutic angiogenesis for ischemic cardiovascular disease. J Mol Cell Cardiol 2001, 33(3):379-393.

3. Rosengart TK, Lee LY, Patel SR, Sanborn TA, Parikh M, Bergman GW, Hachamovitch R, Szulc M, Kligfield PD, Okin PM, Hahn RT, Devereux RB, Post MR, Hackett NR, Foster T, Grasso TM, Lesser ML, Isom OW, Crystal RG: Angiogenesis gene therapy: phase I assessment of direct intramyocardial administration of an adenovirus vector expressing VEGF121 CDNA to individuals with clinically significant severe coronary artery disease. Circulation 1999, 100(5):468-474.

4. Nathan DM, Cleary PA, Backlund JY, Genuth SM, Lachin JM, Orchard TJ, Raskin P, Zinman B: Diabetes Control and Complications Trial/ Epidemiology of Diabetes Interventions and Complications (DCCT/EDIC) Study Research Group: Intensive diabetes treatment and cardiovascular disease in patients with type 1 diabetes. N Engl J Med 2005, 353(25):2643-2653.

5. Adeghate E: Molecular and cellular basis of the etiology and management of diabetic cardiomyopathy: a short review. Mol Cell Biochem 2004, 261(1-2):187-191.

6. Federman DG, Bravata DM, Kirsner RS: Peripheral arterial disease: systemic disease extending beyond the affected extremity. Geriatrics 2004, 59(4):26.

7. Asahara T, Murohara T, Sullivan A, Silver M, van der Zee R, Li T, Witzenbichler B, Schatteman G, Isner JM: Isolation of putative progenitor endothelial cells for angiogenesis. Science 1997, 275(5302):964-967.

8. Vasa M, Fichtlscherer S, Aicher A, Adler K, Urbich C, Martin H, Zeiher AM, Dimmeler S: Number and migratory activity of circulating endothelial progenitor cells inversely correlate with risk factors for coronary artery disease. Circ Res 2001, 89(1):E1-E7.

9. Chen YH, Lin SJ, Lin FY, Wu TC, Tsao CR, Huang PH, Liu PL, Chen YL, Chen JW: High glucose impairs early and late endothelial progenitor cells by modifying nitric oxide-related but not oxidative stress-mediated mechanisms. Diabetes 2007, 56(6):1559-1568.

10. Yue WS, Lau KK, Siu CW, Wang M, Yan GH, Yiu KH, Tse HF: Impact of glycemic control on circulating endothelial progenitor cells and arterial stiffness in patients with type 2 diabetes mellitus. Cardiovasc Diabetol 2011, 10:113.

11. Toyokawa $H$, Matsui $Y$, Uhara J: Promotive effects of far-infrared ray on full-thickness skin wound healing in rats. Exp Biol Med 2003, 228(6):724-729.

12. Capon A, Mordon S: Can thermal lasers promote skin wound healing? Am J Clin Dermatol 2003, 4(1):1-12.

13. Kihara T, Biro S, lkeda Y: Effects of repeated sauna treatment on ventricular arrhythmias in patients with chronic heart failure. Circ J 2004, 68(12):1146-1151.

14. Imamura M, Biro S, Kihara T: Repeated thermal therapy improves impaired vascular endothelial function in patients with coronary risk factors. $J \mathrm{Am}$ Coll Cardiol 2001, 38(4):1083-1088.

15. Lin CC, Liu XM, Peyton K, Wang H, Yang WC, Lin SJ, Durante W: Far infrared therapy inhibits vascular endothelial inflammation via the induction of heme oxygenase-1. Arterioscler Thromb Vasc Biol 2008, 28(4):739-745.

16. Akasaki Y, Miyata M, Eto H, Shirasawa T, Hamada N, Ikeda Y, Biro S, Otsuji Y, Tei C: Repeated thermal therapy up-regulates endothelial nitric oxide synthase and augments angiogenesis in a mouse model of hindlimb ischemia. Circ J 2006, 70(4):463-470. 
17. Yu SY, Chiu JH, Yang SD, Hsu YC, Lui WY, Wu CW: Biological effect of far-infrared therapy on increasing skin microcirculation in rats. Photodermatol Photoimmunol Photomed 2006, 22(2):78-86.

18. Huang PH, Lin CP, Wang CH, Chiang CH, Tsai HY, Chen JS, Lin FY, Leu HB, Wu TC, Chen JW, Lin SJ: Niacin improves ischemia-induced neovascularization in diabetic mice by enhancement of endothelial progenitor cell functions independent of changes in plasma lipids. Angiogenesis 2012, 15(3):377-389.

19. Kilkenny C, Browne WJ, Cuthill IC, Emerson M, Altman DG: Improving bioscience research reporting: the ARRIVE guidelines for reporting animal research. PLOS Biol 2010, 8(6):1000412.

20. Huang PH, Chen YH, Wang CH, Chen JS, Tsai HY, Lin FY, Lo WY, Wu TC, Sata M, Chen JW, Lin SJ: Matrix metalloproteinase-9 is essential for ischemia-induced neovascularization by modulating bone marrowderived endothelial progenitor cells. Arterioscler Thromb Vasc Biol 2009, 29(8):1179-1184

21. Huang PH, Chen JS, Tsai HY, Chen YH, Lin FY, Leu HB, Wu TC, Lin SJ, Chen JW: Globular adiponectin improves high glucose-suppressed endothelial progenitor cell function through endothelial nitric oxide synthase dependent mechanisms. J Mol Cell Cardiol 2011, 51(1):109-119.

22. Huang $\mathrm{PH}$, Huang SS, Chen YH, Lin CP, Chiang KH, Chen JS, Tsai HY, Lin FY, Chen JW, Lin SJ: Increased circulating CD31+/annexin V + apoptotic microparticles and decreased circulating endothelial progenitor cell levels in hypertensive patients with microalbuminuria. J Hypertens 2010, 28(8):1655-1665

23. Takahashi T, Kalka C, Masuda H, Chen D, Silver M, Kearney M, Magner M, Isner JM, Asahara T: Ischemia- and cytokine-induced mobilization of bone marrow-derived endothelial progenitor cells for neovascularization. Nat Med 1999, 5(4):434-438.

24. Fadini GP, de Kreutzenberg S, Albiero M, Coracina A, Pagnin E, Baesso I, Cignarella A, Bolego C, Plebani M, Nardelli GB, Sartore S, Agostini C, Avogaro A: Gender differences in endothelial progenitor cells and cardiovascular risk profile: the role of female estrogens. Arterioscler Thromb Vasc Biol 2008, 28(5):997-1004.

25. Palombo C, Kozakova M, Morizzo C, Gnesi L, Barsotti MC, Spontoni P, Massart F, Salvi P, Balbarini A, Saggese G, Di Stefano R, Federico G: Circulating endothelial progenitor cells and large artery structure and function in young subjects with uncomplicated type 1 diabetes. Cardiovasc Diabetol 2011, 10:88.

26. Li H, Zhang $X$, Guan $X$, Cui $X$, Wang $Y$, Chu H, Cheng M: Advanced glycation end products impair the migration, adhesion and secretion potentials of late endothelial progenitor cells. Cardiovasc Diabetol 2012, 11:46.

27. Aicher A, Heeschen C, Mildner-Rihm C, Urbich C, Ihling C, Technau-lhling $\mathrm{K}$, Zeiher AM, Dimmeler S: Essential role of endothelial nitric oxide synthase for mobilization of stem and progenitor cells. Nat Med 2003, 9(11):1370-1376

28. Thum T, Tsikas D, Stein S, Schultheiss M, Eigenthaler M, Anker SD, PooleWilson PA, Ertl G, Bauersachs J: Suppression of endothelial progenitor cells in human coronary artery disease by the endogenous nitric oxide synthase inhibitor asymmetric dimethylarginine. J Am Coll Cardiol 2005, 46(9):1693-1701.

29. Park JH, Miyashita M, Kwon YC, Park HT, Kim EH, Park JK, Park KB, Yoon SR, Chung JW, Nakamura Y, Park SK: A 12-week after-school physical activity programme improves endothelial cell function in overweight and obese children: a randomised controlled study. BMC Pediatr 2012, 12(1):111.

30. Gryglewski RJ, Palmer RM, Moncada S: Superoxide anion is involved in the breakdown of endothelium-derived vascular relaxing factor. Nature 1986, 320(6061):454-456.

31. Hartel M, Hoffmann G, Wente MN, Martignoni ME, Buchler MW, Friess $H$ : Randomized clinical trial of the influence of local water-filtered infrared A irradiation on wound healing after abdominal surgery. Br J Surg 2006 93(8):952-960

32. Hsu YH, Chen YC, Chen TH, Sue YM, Cheng TH, Chen JR, Chen CH: Far-infrared therapy induces the nuclear translocation of PLZF which inhibits VEGF-induced proliferation in human umbilical vein endothelial cells. PLoS One 2012, 7(1):e30674.

33. Seeger FH, Haendeler J, Walter DH, Rochwalsky U, Reinhold J, Urbich C, Rössig L, Corbaz A, Chvatchko Y, Zeiher AM, Dimmeler S: p38 mitogen-activated protein kinase downregulates endothelial progenitor cells. Circulation 2005, 111(9):1184-1191. doi:10.1186/1475-2840-11-99

Cite this article as: Huang et al.: Far infra-red therapy promotes ischemia-induced angiogenesis in diabetic mice and restores high glucose-suppressed endothelial progenitor cell functions. Cardiovascular Diabetology 2012 11:99.

\section{Submit your next manuscript to BioMed Central and take full advantage of:}

- Convenient online submission

- Thorough peer review

- No space constraints or color figure charges

- Immediate publication on acceptance

- Inclusion in PubMed, CAS, Scopus and Google Scholar

- Research which is freely available for redistribution

Submit your manuscript at www.biomedcentral.com/submit
Ciomed Central 